\title{
How Well Does the PCA3-incorporated Chun Nomogram Perform in Predicting Prostate Biopsy Outcome Among South African Men?
}

The incidence of prostate cancer among South African men is just as significant as it is worldwide [1,2]. Although the role of the prostate cancer antigen 3 (PCA3) assay in predicting biopsy outcome has proven beneficial in a South African context [3], the assessment of its role incorporated into a prostate cancer risk calculator has not yet been explored on the continent of Africa. We aimed to assess the performance of the PCA3-incorporated Chun nomogram [4] and to compare its performance with other contemporary risk calculators.

We prospectively evaluated 107 consecutive South African men that were already scheduled for a 13-core transrectal ultrasound prostate biopsy at two referral centres in the South African capital city, Pretoria. The urine PCA3 assay was always performed prior to biopsy and was evaluated using the Progensa assay (GenProbe, San Diego, CA, USA). Performance of the following four calculators was compared:

- Chun nomogram [4]

- Prostate Cancer Prevention Trial (PCPT) risk calculator [5]

- Updated (PCA3 incorporated) PCPT risk calculator [5]

- European Randomised Study of Screening for Prostate Cancer (ERSPC) risk calculator [6]

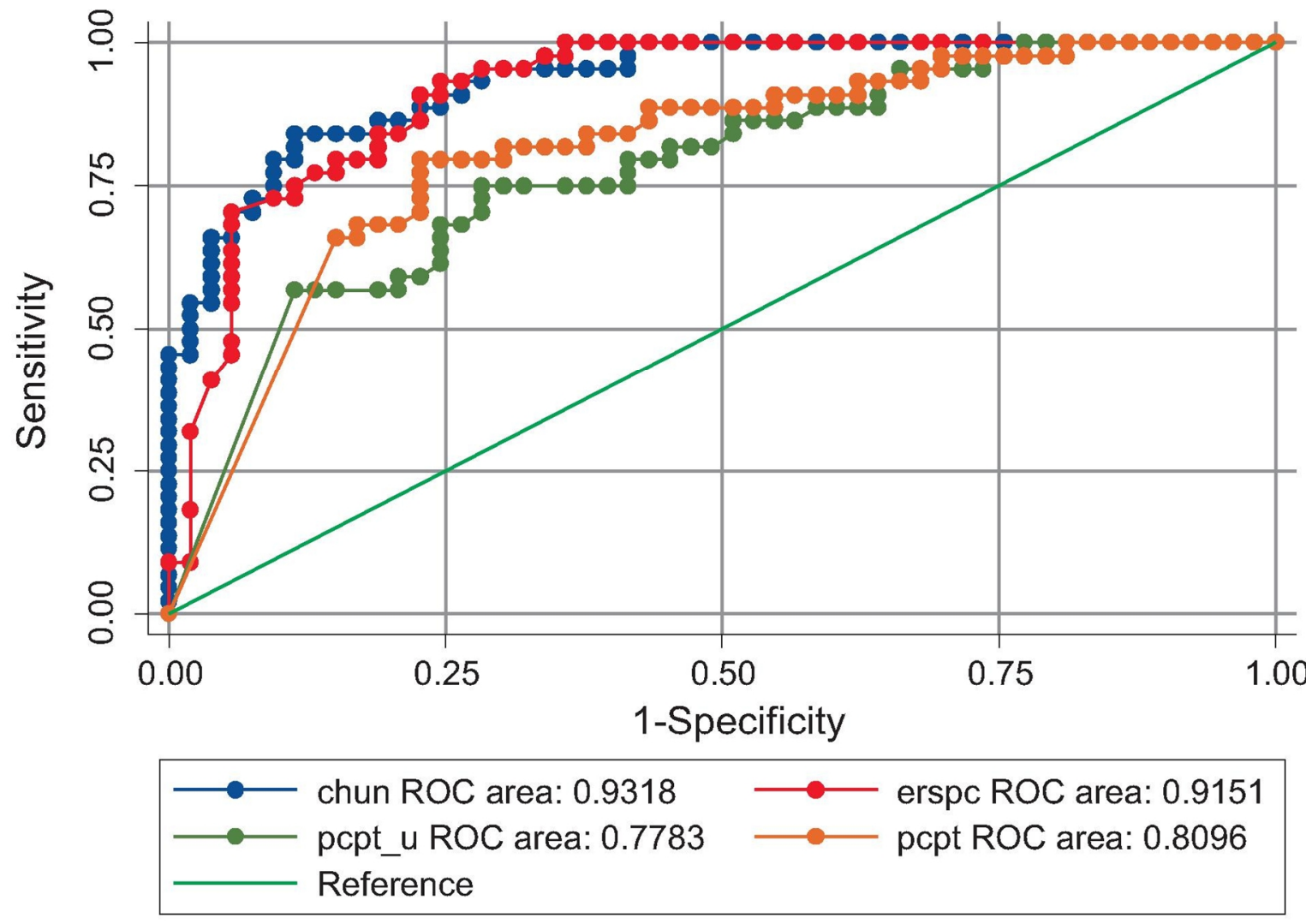

\section{Fig. 1 - Receiver operating characteristic (ROC) curves of the four prostate cancer risk calculators in a South African setting.}

Histologic evidence of cancer in the prostate biopsy specimen was set as the primary end point. The 
discriminating ability of each risk calculator was assessed and compared using the concordance index (cindex). The mean patient age was $67 \mathrm{yr}$. A positive cancer outcome was present in 46 of 107 men, with only 19 of 107 scheduled for a repeat biopsy. Ultimately, 97 patients were assessed because 10 patients were excluded from the comparison data set due to inconclusive PCA3 assay scores (2 of 10) and patient age $<55$ yr $(8$ of 10$)$.

The four calculators had the following c-indexes: Chun nomogram, 0.93 (95\% confidence interval [CI], 0.88-0.97); PCPT, 0.80 (95\% CI, 0.72-0.89); updated PCPT, 0.77 (95\% CI, 0.68-0.86); ERSPC, 0.91 (95\% CI, 0.85-0.97) (Fig. 1).

The Chun nomogram outperformed the other calculators assessed. Because the initial development of the Chun nomogram involved multiple centres in Europe and included both Canada and the United States [4], our findings may further support the universality of this PCA3-incorporated risk calculator.

The PCPT risk calculators do not have a specific option within the racial category for the local black African man, so the option other was used for this racial group. This may have underestimated the risk for a certain racial subset in our study and may explain the poorer performance of the PCPT and updated PCPT risk calculators.

Because the ERSPC risk calculator also performed exceptionally well, the cost-benefit ratio of incorporating the PCA3 assay into a risk calculator may be questioned. The role of a more recent PCA3incorporated, initial-biopsy-specific nomogram [7] may be of added value within the initial biopsy subset of patients in our context. Further evaluation in this regard is needed. For now, the PCA3-incorporated Chun nomogram has outperformed other risk calculators in this setting.

Conflicts of interest: The authors have nothing to disclose.

Acknowledgment statement: We wish to thank IlexSA Medical and Lancet Laboratories (South Africa) for bearing the costs of the PCA3 assay testing. We are grateful to Dr Felix Chun (Hamburg, Germany), for allowing us the access to the actual coefficient variables within the 'Chun Nomogram'. We also wish to thank Farhaad Amod, MSc (Mathematical Statistics), University of Johannesburg, for his statistical support and technical advice.

\section{References}

[1] Center MM, Jemal A, Lortet-Tieulent J, et al. International variation in prostate cancer incidence and mortality rates. Eur Urol 2012;61: 1079-92.

[2] Heyns CF. Is prostate cancer more common and more aggressive in African men? Afr J Urol 2008;14:66-74.

[3] Adam A, Engelbrecht MJ, Bornman MS, et al. The role of the PCA3 assay in predicting prostate biopsy outcome in a South African setting. BJU Int 2011;108:1728-33.

[4] Chun FK, de la Taille A, van Poppel H, et al. Prostate cancer gene 3 (PCA3): development and internal validation of a novel biopsy nomogram. Eur Urol 2009;56:659-68.

[5] Individualized risk assessment of prostate cancer. University of Texas Health Science Center at San Antonio Department of Epidemiology and Biostatistics Web site. http://deb.uthscsa.edu/URORisk Calc/Pages/calcs.jsp.

[6] The prostate cancer risk calculators - including the 'future risk' calculator. SWOP - The Prostate Cancer Research Foundation, Rotterdam, Web site. http://www.prostatecancer-riskcalculator. com/seven-prostate-cancer-risk-calculators.

[7] Hansen J, Auprich M, Ahyai SA, et al. Initial prostate biopsy: development and internal validation of a biopsy-specific nomogram based on the prostate cancer antigen 3 assay. Eur Urol 2013;63: 201-9.

Ahmed Adam ${ }^{\mathrm{a}, * *}$

Samuel O. Manda ${ }^{\mathrm{b}}$

${ }^{a}$ Department of Urology, University of Pretoria, Steve Biko Academic Hospital, Pretoria, South Africa

${ }^{b}$ The Biostatistics Unit, The Medical Research Council, Pretoria, South Africa

*Corresponding author. Department of Urology,

Steve Biko Academic Hospital, Level 7, Bridge C, Room 71207, Pretoria, Gauteng 0001, South Africa.

Tel. +2712 354 1513; Fax: +27123542500.

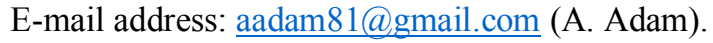

